\title{
Expertengespräch: Komplementäre Onkologie
}

Der Komplementärmedizin wird oft vorgeworfen, sie wirke nicht. Wirkt sie in der Onkologie?

Nagel: Es kommt immer darauf an, was wir unter «wirken» verstehen und in Bezug auf was etwas wirken soll. Die Komplementärmedizin ist dazu da, den Patienten in seinem Tun und in seinem «Self Empowerment» zu stärken - das sollte sie schon leisten, und das leisten viele Verfahren auch.

\section{Was ist der Unterschied zwischen einer klassischen medikamentösen Tumortherapie und den integrativen komplementärmedizinischen Ansätzen?}

Nagel: Die Komplementärmedizin hat nichts mit Tumortherapie zu tun. Es geht darum, den Patienten zu unterstützen. Egal, ob die Ärzte sagen, wir wissen nicht, wie ein bestimmtes Verfahren wirkt, und können auch nichts über die Wirkung sagen - heute entscheiden die Patienten nach ihrem Gefühl, ob ihnen eine Methode hilft und nützt. Ihnen ist es egal, ob die Wirkung wissenschaftlich belegt ist. Für sie ist es die Hauptsache, dass sie das Gefühl haben, von ihr zu profitieren.

Prof. Dr. Gerd Nagel, der Leiter der von ihm ins Leben gerufenen Stiftung Patientenkompetenz in Zürich, und Dr. med. Jutta Hübner, Leiterin Palliativmedizin und komplementäre Onkologie des Klinikums der GoetheUniversität in Frankfurt/Main, sprechen über die Herausforderungen der Komplementärmedizin in der Onkologie.

Die Wirkung ist nicht physiologisch, sondern zielt auf das sehr subjektive Gefühl des Patienten?

Nagel: Das ist nicht nur subjektiv, sondern hängt wesentlich von den Zielparametern ab, die je nach der Methode sehr unterschiedlich sein können. Die Steigerung der Lebensqualität, die oft im Mittelpunkt steht, wenn Patienten Komplementärmedizin wünschen, ist eines von vielen messbaren Kriterien.

Hübner: Die Wirkung von integrativen Methoden ist selbstverständlich konkret, physiologisch und im Prinzip auch messbar. Wenn mich ein Patient fragt, welche integrativen Methoden ich ihm empfehlen kann, rate ich die Methoden anzuwenden,

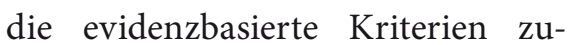
mindest in einem gewissen Umfang erfüllen.

Bei den Ansätzen, in denen das weniger der Fall ist, weil z.B. entsprechende Studien fehlen, kann man es dem Patienten anheimstellen, ob er zu einer bestimmten Methode greifen will. Voraussetzung ist aber, dass kein Risiko damit verbunden ist und die Tumortherapie nicht beeinträchtigt wird. Man muss die Unsicherheit hinsichtlich der Wirkung auch entsprechend kommunizieren und klar sagen: «Wir wissen nicht genau, ob es wirkt, aber Sie können das ausprobieren und sich Ihre eigene Meinung bilden. Es schadet aber nicht.»
Nagel: Der Patient muss deutlich machen, was er eigentlich will. Er kann nicht nur sagen, dass er Komplementärmedizin möchte - die Frage muss immer die nach dem Ziel sein, das er mit der Behandlung erreichen möchte. In der Komplementärmedizin gibt es der Patient im Optimalfall vor. Deshalb muss der Arzt mit dem Patienten darüber sprechen, was dieser von der Behandlung erwartet. Der Dialog über dieses Thema ist für den Erfolg und die Zufriedenheit des Patienten sehr wichtig.

\section{Ein Arzt sollte also mit seinen} Patienten über deren Ziele der Behandlung mit komplementären Verfahren sprechen?

Hübner: Nicht nur bei der Behandlung mit komplementären Verfahren. Wenn wir wirklich integrativ denken und entsprechend behandeln, müssen wir auch bei der konventionellen Therapie die Ziele des Patienten stärker im Auge haben. Die Palliativmedizin ist ein schönes Beispiel, denn dort geht es darum, eine Behandlung zu wählen, die sich an den Vorstellungen des Patienten über sein Leben mit der Krankheit orientiert. Ich glaube, diesen Ansatz brauchen wir auch in der primären Onkologie. Dort können die Behandlungsziele des Patienten von denen abweichen, die der Onkologe hat. Wichtig ist, dass Arzt und Patient über die Ziele der Behandlung sprechen und so die Grundlage für ein gutes Arzt-Patienten-Verhältnis legen.

\section{KARGER}

Fax +49 7614520714 Information@Karger.de www.karger.com 


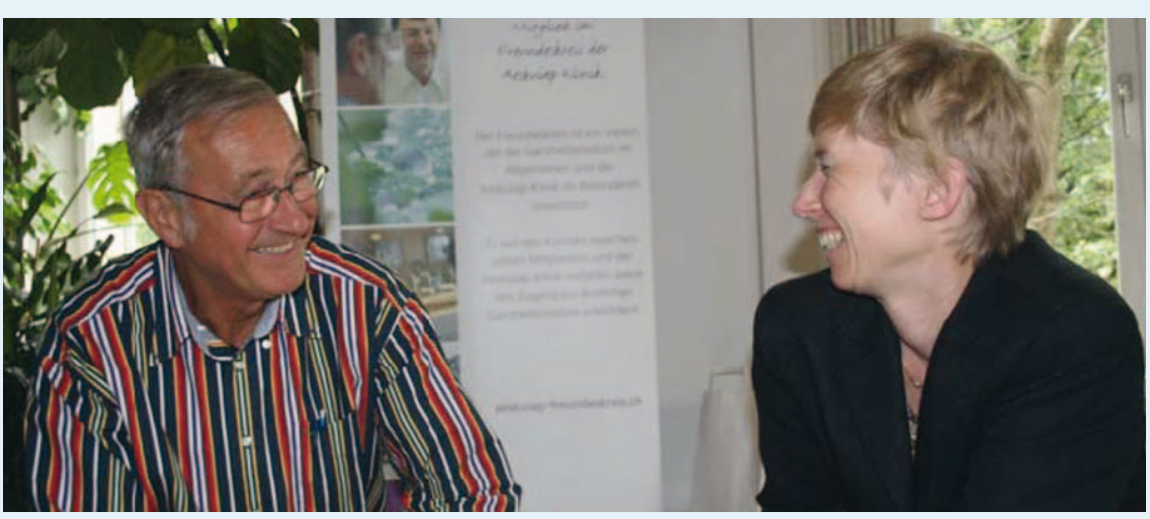

Prof. Dr. med. Gerd Nagel

(g.nagel@patientenkompetenz.ch) hat sein berufliches Leben als Onkologe und Krebsforscher an den Universitäten Basel, Brüssel, New York, Göttingen und Freiburg verbracht. Im Jahr 2003 wurde er von der Stiftung Pro Europa für sein ganzheitliches Medizinmodell mit dem Europäischen WissenschaftsKulturpreis geehrt. Heute leitet er die von ihm ins Leben gerufene Stiftung Patientenkompetenz (www.patientenkompetenz.ch) in Zürich.

«Die Komplementärmedizin ist dazu da, den Patienten in seinem Tun und in seinem "Self Empowerment» zu stärken - das sollte sie schon leisten, und das leisten viele Verfahren auch.»

Im Moment gibt der Arzt die Marschrichtung vor: Er bestimmt die Zielsetzung der Behandlung in der konventionellen Medizin, und der Patient muss ihm folgen.

Hübner: Der Arzt geht davon aus, dass er schon von vornherein genau weiss, was das Ziel des Patienten bei der Behandlung seiner Krebserkrankung ist. Fragt er ihn hingegen nach seinen Vorstellungen, ist er bisweilen höchst erstaunt, wenn der Patient sich etwas ganz anderes von der Therapie verspricht.

Heute gibt es in der Regel über das Ziel der Behandlung keinen Gedankenaustausch zwischen Arzt und Patienten. Über die Behandlung entscheiden die medizinischen Fachleute - im Tumorboard wird eine Therapieempfehlung abgegeben, die auf den Zielen der Experten beruht.

\section{Dr. med. Jutta Hübner}

(jutta.huebner@kgu.de) ist Sprecherin des Arbeitskreises komplementäre Onkologie in der Deutschen Krebsgesellschaft und Leiterin Palliativmedizin und komplementäre Onkologie des Klinikums der Goethe-Universität in Frankfurt. Ihr neuestes Patientenbuch, «Aloe, Ginkgo, Mistel und Co. Ergänzende Wirkstoffe in der Krebsbehandlung», ist im Herbst erschienen.

"Ich hoffe, dass wir in 20 Jahren tatsächlich bei der integrativen Medizin angekommen sind, die nicht mehr in den getrennten Konstrukten von Schulmedizin und komplementärer Medizin denkt.»

Bedeutet das, dass die Kommunikation zwischen Arzt und Patient anders werden muss? Der Patient muss mit seinen Wünschen ernst genommen werden.

Nagel: Auf alle Fälle. Die Wünsche des Patienten werden heute ernster genommen als früher. Als ich vor fast 50 Jahren mit der Onkologie angefangen habe, war es uns verboten, den Patienten ihre Diagnosen zu sagen. Folglich konnte man gar nicht kommunizieren. Das ist jetzt ganz anders, weil es Pflicht ist, dem Patienten den Befund zu eröffnen. Deshalb wird heute ganz anders kommuniziert. Man muss klar feststellen, dass heute zum Teil hervorragend mit den Patienten geredet wird, aber leider zum Teil auch noch furchtbar schlecht kommuniziert wird.

Schlecht ist die Kommunikation nach der Holzhammermethode - gar nicht $\mathrm{zu}$ reden oder einfach im falschen Moment. Man kann mit einem
Patienten nicht über die Konsequenzen seiner Krebserkrankung reden, wenn man ihm gerade die Diagnose mitgeteilt hat. In dieser Situation ist er blockiert, hat einen Schock und denkt nicht rational. Er bewegt sich auf einer irrationalen Ebene. Kommunikation in einem solchen Augenblick ist die Ursache für Missverständnisse. Kein Wunder, dass der Patient später sagt: «Mein Arzt hat nie mit mir geredet» und der Doktor überzeugt ist: «Ich hab' stundenlang mit dem Patienten gesprochen.» Stimmt - allerdings im falschen Moment.

Hat die vermehrte Kommunikation mit den Patienten und auch ihre bessere Informiertheit zu einem grösseren Bedarf an Komplementärmedizin geführt?

Hübner: Ich habe den Eindruck, dass durch mehr Offenheit und mehr Kommunikation das Thema Komplementärmedizin häufiger angesprochen wird. $\mathrm{Ob}$ dadurch ein grösserer Bedarf entstanden ist oder ob wir den Bedarf vorher nur nicht wahrgenommen haben, weiss ich nicht.

Nagel: Die Kommunikation ist eine grosse Chance für die Komplementärmedizin. Ich glaube, dass die Nichtkommunikation oder die Verweigerung des Gesprächs über Komplementärmedizin die Patienten in die falsche Richtung treibt, nämlich zu unseriösen Methoden und in die Arme von Scharlatanen. Wenn man mit ihnen ein sehr genaues Gespräch über die Möglichkeiten und Ziele der Komplementärmedizin führt, sinkt die Nachfrage nach den entsprechenden Methoden. Der Grund ist klar: Die utopischen Erwartungen sind nicht mehr da.

Hübner: Deshalb müssen wir die Fragen der Patienten nach ihren Möglichkeiten, um besser mit der Krankheit umzugehen, sehr seriös beantworten. Ich erlebe, dass Patienten, die wir intensiver beraten, mitunter viel weniger 
aus den verschiedenen Töpfen der Komplementärmedizin nehmen. Sie sind bei der Wahl der Behandlung, die sie dann wählen, sehr fokussiert. Sie sind mit ihren Entscheidungen auch glücklicher als die, die mit dem riesigen Angebot der Komplementärmedizin ohne ärztliche Beratung alleine gelassen sind. Die modernen Medien wie das Internet sind ein gefährliches Instrument für die Patienten. Sie haben keine Kriterien, an denen sie sich orientieren können, um die Informationen dort bewerten zu können.

Nagel: Viele Patienten sind tatsächlich überinformiert und einfach verwirrt. Das verunsichert zur Diagnose zusätzlich, und die Patienten sind orientierungslos. Die Aufgabe der Mediziner ist es, ihnen in Gesprächen die Orientierung wieder zu geben. Sie müssen von ihrem Arzt erfahren, welche Methoden wichtig und richtig sind - und welche nicht. Wenn die Patienten wieder orientiert sind, wenn sie wieder wissen, wo sie stehen, verlangen sie gar nicht mehr so viele verschiedene komplementäre Methoden. Sie wollen dann gar nicht mehr alles Mögliche ausprobieren, sondern konzentrieren sich auf Weniges und Bewährtes.

\section{Der Patient braucht auch in der Komplementärmedizin die Leitung durch «seinen» Schulmediziner?}

Hübner: Es braucht die Kompetenz in der integrativen Medizin bei den Onkologen. Im Moment erleben wir eine Zwischenstufe in der Entwicklung hin zur integrativen Medizin, die ich gefährlich finde. Im Gegensatz zur älteren Generation der Onkologen ist die jüngere bezüglich komplementären und alternativen Methoden oft sehr liberal eingestellt. Sie überlässt den Patienten die Auswahl; allerdings häufig, ohne sie zu beraten. Die Patienten dürfen vom Arzt aber eine fundierte Meinung und auch Empfehlungen erwarten. Ich habe das Gefühl, dass wir bei der Akzeptanz der Kom- plementärmedizin fast von einem Extrem in das andere kippen.

Der Ansatz, den Sie in Ihren Büchern über die Komplementärmedizin vertreten, ist ein sehr mechanistischer. Sie führen verschiedene Verfahren der Komplementärmedizin auf den pharmakologischen Weg zurück. Stört das nicht viele, die in der integrativen Medizin gerade einen anderen Ansatz, einen nicht wissenschaftlichen, suchen?

Hübner: Ich glaube, dass wir mit einem naturwissenschaftlichen Ansatz bei den «Schulmedizinern» und bei den Patienten eine gute Akzeptanz finden. Er vermittelt den Patienten Orientierung, weil die Methoden aufgrund klarer und nachvollziehbarer Kriterien beurteilt werden.

Entscheidend ist, dass sich die integrative Medizin auf dem Boden der Wissenschaftlichkeit bewegt. Allerdings muss man teilweise methodische Einschränkungen bei der Untersuchung ihrer Wirksamkeit machen. Bei manchen Evaluationen und Fragestellungen eignet sich der Ansatz der randomisierten, doppelblinden, Placebo-kontrollierten Studie nicht. Dieses Phänomen kennen wir z.B. auch aus der Psychoonkologie. In diesen Fällen muss die Wirksamkeit mit einem anderen ebenfalls aussagekräftigen Ansatz untersucht werden.

Für mich gibt es den Gegensatz zwischen der integrativen Medizin und der Schulmedizin nicht. Es gibt nur die Trennung der wissenschaftlichen Medizin von der Scharlatanerie: die seriöse Medizin auf der einen Seite und die unseriösen Methoden auf der anderen. Die Entwicklung letzterer wird zum Teil aus ökonomischen Interessen forciert. Deren Verfechter wollen von den Ängsten und Hoffnungen von Kranken profitieren und haben zum grossen Teil auch gar kein Interesse daran, dass ihre Wirksamkeit wissenschaftlich untersucht wird.
Nagel: Für den Erfolg der Behandlung ist meiner Ansicht nach die Kompetenz des ärztlichen Beraters sehr wichtig. Wenn ein Patient ein Orientierungsgespräch wünscht und dies ein 25-jähriger Arzt durchführt, der gerade sein Staatsexamen gemacht hat, scheitert es oft. Das liegt nicht an der medizinischen Kompetenz, sondern an den sehr unterschiedlichen Lebenshintergründen und -erfahrungen der beiden. Der junge Arzt findet bei einem reifen Patienten einfach keine Akzeptanz - er versteht oft auch die Patientensprache gar nicht. Die Behandlung scheitert an der falschen Sozialkompetenz.

\section{Wie gross wird die Bedeutung} der Komplementärmedizin in der Onkologie in 10 Jahren sein?

Hübner: Wenn man den Zeitrahmen auf 20 Jahre verlängert, hoffe ich, dass wir dann tatsächlich bei der integrativen Medizin angekommen sind, die nicht mehr in den getrennten Konstrukten von Schulmedizin und komplementärer Medizin denkt. Ich wünsche mir, dass wir dann mit einem ganzheitlichen medizinischen Modell arbeiten, in dem die integrative Medizin gleichbedeutend mit wissenschaftlicher Medizin ist. Und dass wir uns als Komplementärmediziner ganz klar von dem abgrenzen, was an Methoden unseriös, unwissenschaftlich und kontraproduktiv ist. Dass wir das innerhalb der nächsten 10 Jahre erreichen können, glaube ich nicht.

Nagel: Dieser Wandel wird eine Frage von Generationen sein. Heute findet eine entsprechende Ausbildung der Studentinnen und Studenten an den Universitäten gar nicht statt. Sie werden an den Hochschulen ganz strikt nach dem herkömmlichen Konzept dessen geprägt, was wir heute unter der Schulmedizin verstehen. Die wichtigen Ansätze einer integrativen Medizin müssen sie sich später in der täglichen Praxis aneignen. Das muss sich ändern. 
Eine junge Ärztin oder ein junger Arzt lernt den integrativen Ansatz heute quasi im Crashkurs beim Umgang mit den Patienten und ohne ausreichende Ausbildung?

Nagel: Die integrative Kompetenz erwirbt man sich aus Erfahrungswerten, die man mit der Zeit macht. Ich glaube, dass die Hausärzte, die schon lange in ihrer Praxis tätig sind, längst integrative Medizin betreiben - auch wenn sie es selber nicht so formulieren würden. Die Mehrheit der Praktiker behandelt integrativ.

Hübner: Die Praktiker betreiben die integrative Medizin intuitiv und basierend auf ihrer grossen Erfahrung. Ich glaube, dass sie sich gar nicht bewusst sind, wie gut sie ihre Patienten ganzheitlich behandeln.

Für mich stellte sich gerade die Frage nach dem Stellenwert des integrativen Ansatzes in der Ausbildung von Studierenden im Moment konkret bei der Vorbereitung der Palliativ-Vorlesung an der Universität Frankfurt. Sollen sie den ganzen Wissenskatalog verinnerlichen, der von der entsprechenden Fachgesellschaft verabschiedet wurde, der aber zum Teil veraltet sein wird, wenn sie in einigen Jahren zum ersten Mal Verantwortung für Patienten haben? Oder ist es wichtiger, dass sielernen, wie man mit einem $\mathrm{Pa}$ tienten umgeht und mit ihm spricht? Ich tendiere zu letzterem.

\section{War die komplementäre Medizin} schon immer ein Anliegen von Ihnen?

Nagel: Für mich war Medizin immer eine Mischung aus Fachkompetenz und Sozialkompetenz. Der gute Arzt hat und braucht beides, um seine Patienten angemessen behandeln $\mathrm{zu}$ können. Ein entscheidender Punkt, der meistens vernachlässigt wird, ist tatsächlich die Sozialkompetenz des Arztes, denn sie erlaubt ihm die Wahrnehmung der komplementären Wirklichkeit des Patienten. Mit ihrer Hilfe kann er versuchen, ihn zu verstehen, und ihn im Gespräch dort abholen, wo er steht. Das ist die Grundlage für eine gute Beratung über die Möglichkeiten und Grenzen der komplementären Medizin bei der Behandlung seiner Krebserkrankung.

In der Schulmedizin herrscht immer noch eine Skepsis gegenüber den Methoden der integrativen Medizin vor. Worin liegt der Grund dafür?

Hübner: Ich glaube, das hat vor allem damit zu tun, dass wir im Alltag die Begriffe «komplementär» und «alternativ» verschmelzen. Das sollten wir nicht tun, weil es die Grenze zwischen wissenschaftlich evaluierbaren und getesteten Methoden und unseriösen und unklaren Ansätzen verwischt.

Historisch gesehen, hat es oft den Übertritt von Methoden aus der Naturheilkunde oder Erfahrungsheilkunde in den Kanon der Schulmedizin gegeben. Eines der klassischen Beispiele ist die Verwendung von Digitalis, die in der Erfahrungsheilkunde entwickelt wurde und heute eine schulmedizinische Therapie ist. Die gleiche Entwicklung werden wir mit anderen substanzgebundenen Anwendungen der Komplementärmedizin in Zukunft auch sehen - sie werden Teil dessen, was wir heute als Schulmedizin bezeichnen.

Nagel: Bei der heutigen Trennung von Schul- und Komplementärmedizin verkennt man, dass die Schulmedizin sich intensiv aus dem Fundus der Natur- und Erfahrungsheilkunde alimentiert hat. Neben dem Beispiel Digitalis gibt es eine ganze Reihe von weiteren, die man heute als Standardverfahren betrachtet. Sind sie erst einmal etabliert, werden sie als Methode der Erfahrungsmedizin gar nicht mehr wahrgenommen. Die ganze Rehabilitationsmedizin, die als Schulmedizin gilt, besteht aus Ansätzen und Verfahren, die aus der Erfahrungsmedizin stammen. Sie basieren darauf, dass an die Regenerationskraft des Menschen appelliert wird und sie gestärkt wer- den soll. Andere Beispiele für Disziplinen der Schulmedizin, die Methoden aus der Natur- und Erfahrungsheilkunde übernommen haben, sind etwa die Psychoonkologie und die Physiotherapie - aber auch die Pflege gehört dazu.

\section{Folglich ist es eine Frage der \\ Konvention, was man als \\ Schulmedizin und was man als \\ Komplementärmedizin betrachtet?}

Nagel: Dies basiert auf Traditionen, die im Laufe der Zeit gewachsen sind. Wenn einmal erst der Konsens herrscht, dass z.B. Mikronährstoffe in den Bereich der Komplementärmedizin, Alternativmedizin oder Paramedizin gehören, dann wird das so gelehrt und von den Studentinnen und Studenten auch geglaubt.

Hübner: Eine grosse Schwierigkeit in der Diskussion ist es, dass die Komplementärmedizin von aussen betrachtet oft als ein homogenes Feld wahrgenommen wird. Das ist sie aber nicht - unter dem Begriff werden die unterschiedlichsten Ansätze und Verfahren subsumiert. Sie unterscheiden sich zum einen methodisch und zum anderen nach der wissenschaftlichen Grundlage, auf der ihre Wirkung beurteilt wird. Sie reichen von hochtechnischen Verfahren, die gar nichts mit Naturheilkunde zu tun haben, bis hin zur Erfahrungsmedizin und zur Phytotherapie. Es gibt auf diesem Feld auch eine unterschiedliche Bereitschaft, die Methoden testen und wissenschaftlich überprüfen $\mathrm{zu}$ lassen. Für die Elektroverfahren beispielsweise, die mit der Etikette der Komplementärmedizin vertreten werden, gibt es keine publizierten Studien. Es gibt wirtschaftliche Gründe, warum das Interesse, die Methoden wissenschaftlich prüfen zu lassen, gering ist. Bei anderen Verfahren, wie etwa in der Phytotherapie, ist das Interesse, die Wirksamkeit wissenschaftlich zu belegen, sehr gross.

Interview: Oliver Klaffke

Im Fokus: Komplementäre Onkologie 